\title{
ATMOSPHERIC ANGULAR MOMENTUM AND ITS RELATION TO THE LENGTH OF DAY
}

\author{
R. A. Hull, D. D. McCarthy, C. H. Schwartz \\ U.S. Naval Observatory \\ Washington, D.C. 20392 USA
}

\begin{abstract}
The U.S. Naval Observatory is responsible for the determination and prediction of UT1-UTC. An investigation was begun to determine if atmospheric angular momentum (AAM) data could be useful in the National Earth Orientation Service (NEOS) combined solution and in the prediction of UT1-UTC. The investigation found AAM data to be useful possibly in the combined solution, but predictions of UT1-UTC were adversely affected when predictions of AAM data were introduced.
\end{abstract}

\section{INTRODUCTION}

This investigation examined the differences between astronomical length of day (LOD) derived from UT1-UTC and the length of day derived from changes in atmospheric angular momentum. Specific areas which were addressed included

1) Smoothing of meteorologically derived LOD,

2) Comparison between AAM wind solutions up to 50 millibar and wind solutions up to 100 millibar levels,

3) Comparison of a wind and pressure solution of AAM with a "wind only" solution for AAM,

4) Investigation of errors in meteorological predictions out to ten days,

5) Introduction of AAM data in the NEOS combination solution,

6) Introduction of AAM data in NEOS prediction of UT 1-UTC out to ten days.

While AAM is known to affect Earth rotation parameters about the three Earth axes $\left(0^{\circ}, 0^{\circ}\right),\left(0^{\circ}, 90^{\circ} \mathrm{E}\right),\left(90^{\circ} \mathrm{N}\right)$ we only studied the effect that $X_{3}$ (the axial effective angular momentum function of the atmosphere) has on the $90^{\circ} \mathrm{N}$ axis and its relation to LOD. This study was also limited to correlations with periods less than one year. We have not, at the present time, studied $X_{1}$ and $X_{2}$ (the equatorial effective angular momentum functions) and their effects on the other axes.

Three sets of data, one astronomical and two observed meteorological sets, were used in the analysis of LOD and AAM. The astronomical data set was the NEOS combination solution utilizing data from various techniques and instruments including Doppler, PZT's, VLBI (IRIS), Satellite Laser Ranging, and the connected 
element interferometer in Green Bank, West Virginia. The astronomical data are unsmoothed and thus contain the high frequency information that might be expected to be correlated with the unsmoothed meteorological data.

The observed meteorological data sets were obtained from the National Meteorological Center (NMC, USA). One is received directly while the other is from the NMC provided through the National Geodetic Survey (NGS). The two AAM data sets are different in their format, but, more importantly, represent different ways of calculating AAM. What is called the "NGS data set" is placed in a file on the G.E. MARK III computer under the file name of "WINDY *". This data set contains AAM data for the Northern and Southern Hemispheres, with and without the inverted barometer model data, integrals for pressures and winds up to the 50 millibar level, as well as all three momentum functions about the Earth axes. Unfortunately this data base is only updated once per month and thus provides only an historical record.

The second meteorological data set comes directly from the National Meteorological Center via a computer link with an automated office system. A wind solution up to 100 millibars is provided daily for the previous day's observed momentum. This is not a complete $X_{3}$ solution but a partial solution of AAM with wind data only and uses data only to a pressure of a 100 millibars. Also given in this data set are predictions up to 10 days in the future for the partial $X_{3}$ solution.

\section{CORRELATION OF DATA SETS}

In examining these three data sets and their correlations, it was hoped that it might be possible to incorporate AAM data in the solution for length of day. A systematic error model of the form:

$$
L O D=A+B(A A M)+C \sin (2 \pi t+p)
$$

was fit to the NMC data where $t$ represents the fraction of the year and $p$ is a phase. No attempt to filter the data was made, but this may be desirable in the future. An expression as given in (1) was derived for each AAM data set. We then used this function in the prediction and combination programs in an attempt to use AAM meteorological data as a technique for calculating LOD.

The differences between a full $X_{3}$ solution (pressures and winds) and a partial $X_{3}$ (wind only) data set were also investigated. We found a significant difference between the full and partial solutions. Basically, the full $X_{3}$ solution from NGS had a statistically significant better correlation with the NEOS data set compared to the partial NMC data set.

To see if this significance was due to the addition of pressures in the NGS data set or if it was just due to the different integration altitudes for the meteorological data, the wind data from NGS (50 millibar) and only the wind data from NMC (100 millabar) were studied. A somewhat better correlation was found for the 50 millibar data than the 100 millibar data with the astronomical AAM. The effect of an inverted barometer correction was also studied. This correction takes into account the response of oceans to surface pressure changes. No significant improvement in using the inverted barometer model correction to $X_{3}$ was found for the high frequency components.

In spite of the finding that the NGS data set for a complete $X_{3}$ solution was significantly better correlated with the NEOS astronomical LOD, it is necessary to use the NMC data set because it is currently the only source of timely AAM information. For AAM data to be incorporated in NEOS predictions and combination solutions, updates must be available at least once per week. The NGS 
data set is only accessible at the end of the month.

Thus, partial $X_{3}$ values for AAM data were installed in the instrument data base. The residuals of these data compared to the other contributing instruments were examined and a statistical weight determined. We then reprocessed 45 weeks of data in the combination program to see how useful AAM data could be for this application. Smoothing the data with a 3-day half-maximum Gaussian filter was found to be appropriate. While AAM data actually provided the lowest weight in the combination solution, they did contribute more than some specific instruments at times. Recall also that these AAM data were not the complete $X_{3}$ solution. We could assume that AAM might provide more weight in the solution if weekly access to the complete $X_{3}$ solution were possible.

Finally, the NMC predictions of AAM were examined. In analyzing over 300 days of predictions it was found that the errors in NMC predictions of AAM were of the order of $\pm 0.2 \mathrm{~ms}$ in time out to ten days. Unfortunately, though, the RMS between the 0 hour observed partial $X_{3}$ solution and the astronomical data was of the order of $\pm 2 \mathrm{~ms}$. From this experimentation we found that predictions of LOD are actually hindered by the introduction of the AAM predictions.

In future studies, modeling of the AAM excitation functions and different types of filtering will be examined. It would also be of interest to examine the differences between 0 and 12 hour meteorological data and how they correlate with LOD.

\section{REFERENCE}

Rosen, R. D., Salstein, D. A., 1983, Variations in Atmospheric Angular Momentum on Global and Regional Scales and Length of Day, J. Geophys. Res. 88 , 5451-5470. 